\title{
Talsperrensicherheit - eine Aufgabe für Generationen
}

\section{Aufleger}

Online publiziert: 4. Juni 2018

(C) Der/die Autor(en) 2018

Zusammenfassung Talsperren sind elementar für das Funktionieren wichtiger Infrastrukturen. Die Überwachung von Talsperren ist von großer Bedeutung für die Sicherheit der unterhalb liegenden Siedlungsräume. Es ist notwendig, die hierfür erforderlichen Prozesse in transparenter und nachhaltiger Weise $\mathrm{zu}$ organisieren. Jede einzelne Talsperre ist ab dem Zeitpunkt ihrer Erstellung in zuverlässiger Form $\mathrm{zu}$ überwachen und regelmäßig dem aktuellen Stand der Technik anzupassen. Im Gegensatz zu vielen anderen Infrastrukturen können Talsperren in der Regel nicht mehr abgebaut bzw. zurückgebaut werden. Die Talsperrenüberwachung ist daher eine Aufgabe, eine Herausforderung, aber auch eine Chance für Generationen. Ingenieure und Ingenieurinnen mit fundierten Kenntnissen in der Talsperrentechnik werden hierfür gebraucht.

\section{Dam safety-a task for generations}

Abstract Dams are critical for the functioning of important infrastructures. The surveillance of dams is of great importance for the safety of the areas downstream. It is necessary to organize the necessary processes in a transparent and sustainable way. Each dam has to be reliably surveyed from the time of its construction. It has to be regularly adapted to the current state of the art. In contrast to many other infrastructures, typically dams cannot be removed again. Therefore, dam surveillance has to be considered as a task, a challenge and a chance for generations. Engineers with sound knowledge in the dam technology are needed for this purpose.

Univ.-Prof. Dr.-Ing. M. Aufleger (殴) Arbeitsbereich Wasserbau, Institut für Infrastruktur, Universität Innsbruck, Technikerstraße 13, 6020 Innsbruck, Österreich

markus.aufleger@uibk.ac.at

\section{Die Sicherheit von Talsperren}

Talsperren sind von elementarer Bedeutung für unsere Gesellschaft. In ihren Stauräumen kann das aus dem natürlichen Einzugsgebiet und aus Überleitungen stammende Wasser zwischengespeichert werden. Talsperren dienen dem Hochwasserschutz, der Bereitstellung von Brauch- und Trinkwasser, der Bewässerung und zur Erzeugung von erneuerbarer Energie aus Wasserkraft. Die Absperrbauwerke der Talsperren stauen das Wasser mitunter sehr weit über das Niveau des natürlichen Gewässers.

Talsperren bestehen unter anderem aus dem Stauraum, einer Hochwasserentlastungsanlage und einem oder auch mehreren Absperrbauwerken. Absperrbauwerke sind entweder Staumauern (aus Beton) oder Staudämme (überwiegend oder vollständig aus Erdbaustoffen). Absperrbauwerke von Talsperren gehören zu den größten Bauwerken weltweit. Große Talsperren besitzen aufgrund der mitunter sehr großen und hoch über dem natürlichen Talboden gespeicherten Wassermassen ein bedeutendes Gefahrenpotenzial.

Talsperren werden zwar häufig unter der Vorgabe einer Lebenszeit oder einer theoretischen Betriebsdauer (z. B. 50 Jahre) geplant und gebaut. Die Erfahrungen der letzten 100 Jahre zeigen jedoch eindrucksvoll, dass Talsperren in der Regel weit über diese ursprünglichen Annahmen hinaus Bestandteil der Infrastruktur und der Landschaft bleiben. Dies hängt mit dem unmittelbaren Nutzen der Anlage, aber auch sehr stark mit den durch einen Aufstau verbundenen hydraulischen und morphologischen Prozessen (insbesondere den Verlandungen im Stauraum) zusammen. Der Rückbau von Talsperren ist meist keine ernsthafte Option. Der Betrieb der Talsperren und insbesondere auch die Überwachung der zughörigen Absperrbauwerke sind daher Aufgaben für Generationen. Hierfür werden ständig fachkundige Ingenieurinnen und Ingenieure benötigt!
Die Anforderungen der Gesellschaft an die Sicherheit von Talsperren sind naturgemäß sehr hoch. Das Risiko des Versagens wesentlicher Anlagenteile von Talsperren muss so klein gehalten werden, dass zu keiner Zeit die Siedlungsräume unterhalb der Absperrbauwerke in der Gesellschaft subjektiv mit einer erhöhten Gefährdung verbunden werden. Für dieses Gefühl wiederum sind unter anderem die Anzahl und der Umfang der tatsächlich passierten Versagensfälle, aber auch deren mediale Aufbereitung von großer Bedeutung. Erheblichen Einfluss auf die Wahrnehmung der potenziellen Gefährdung durch Talsperren hat insbesondere auch der Umgang des Betreibers und der zuständigen Behörden mit diesem Thema. Während noch vor einigen Jahren ein Verschweigen bzw. ein Ignorieren des verbleibenden Versagensrisikos in manchen Regionen Realität war, erhöht eine transparente und realistische Bewertung der Talsperrensicherheit das tatsächliche Sicherheitsniveau und am Ende auch das Vertrauen der modernen Gesellschaft in die Infrastruktur. Die Menschen können durchaus damit umgehen, dass Brückenbauwerke, Verkehrssysteme, Industrieanlagen und auch Talsperren grundsätzlich mit einem Versagensrisiko versehen sind. Wichtig sind Transparenz und Information. Die fortschreitende Digitalisierung bietet hier grundsätzlich große Möglichkeiten. Die Notwendigkeit des Schutzes kritischer Infrastrukturen aufgrund diffuser Bedrohungslagen durch den weltweiten Terrorismus muss in diesem Zusammenhang jedoch auch Beachtung finden.

Das tatsächlich vorhandene Sicherheitsniveau einer Talsperre ist schwierig zu bestimmen. Es ist nicht möglich, die Versagenswahrscheinlichkeit einer Talsperre bzw. des Absperrbauwerks im Sinne eines Zahlenwerts verlässlich zu ermitteln. Für die tatsächliche Sicherheit einer Talsperre sind verschiedenste Aspekte von großer Bedeutung. Sechs besonders wichtige Themen sind (vgl. auch Abb. 1): 


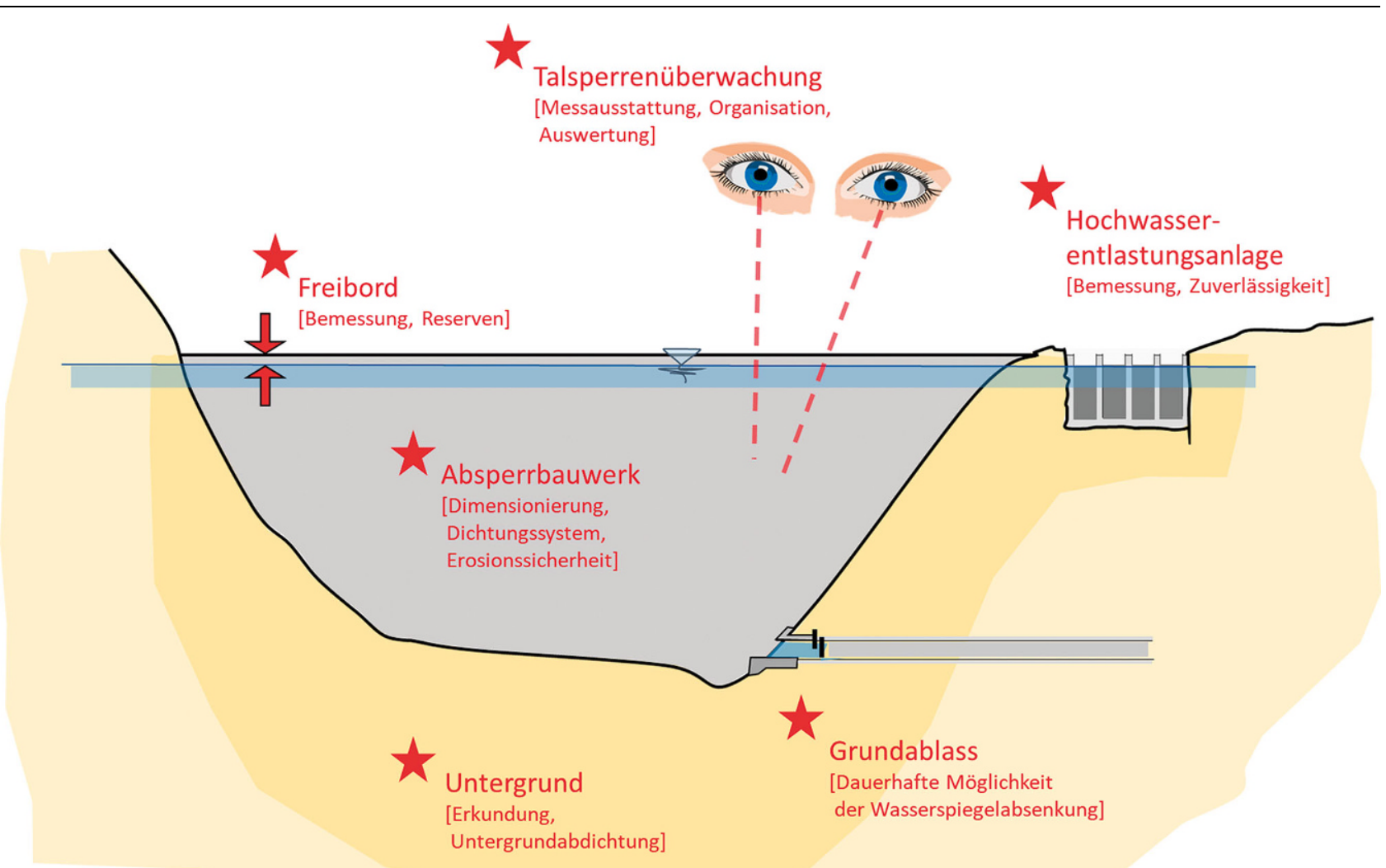

Blick vom Oberwasser auf das Absperrbauwerk einer Talsperre

\section{Abb. 1 Wichtige Aspekte der Sicherheit von Talsperren (Überblick)}

- Die grundsätzliche Dimensionierung des Absperrbauwerks einschließlich des Dichtungssystems unter besonderer Beachtung seiner systembedingten Reserven und der Sicherheit gegenüber inneren Erosionsvorgängen (bei Staudämmen).

- Die Kenntnis der tatsächlichen Untergrundverhältnisse und die Dimensionierung sowie die Ausführung der Untergrundabdichtung.

- Die Leistungsfähigkeit, Ausführung, Verklausungssicherheit und der bauliche Zustand der Hochwasserentlastungsanlage unter besonderer Beachtung der Zuverlässigkeit des Öffnens etwaig vorhandener Verschlüsse.

- Der Freibord als Maß zwischen dem höchsten Stauziel und der Krone des Absperrbauwerks unter Beachtung der Lage der Oberkante des Dichtungssystems.

- Ein betriebssicherer Grundablass, der langfristig in der Lage bleibt, den Wasserspiegel im Stauraum in vergleichsweise kurzer Zeit deutlich abzusenken.
- Die Talsperrenüberwachung als zentrales und unersetzliches Element der Sicherheitsphilosophie einschließlich der daraus resultierenden Anpassungs- und Sanierungsmaßnahmen.

Angesichts der Erheblichkeit des Eingriffes beim Bau von Talsperren und der aufgrund der üblicherweise fehlenden Option des Rückbaus zwingend absehbaren Notwendigkeit späterer Anpassungen liegt es in der Verantwortung der Planer und Betreiber, die Talsperren und ihre Absperrbauwerke von Anfang an mit ausreichenden „zusätzlichen“ Sicherheiten zu versehen. Hierbei spielen der Freibord und die Breite der Krone des Absperrbauwerks jeweils eine ganz besondere Rolle:

- Der Freibord wird üblicherweise unter Beachtung der möglichen Windwellenwirkungen bemessen. Je nach Lastfall und regionaler bzw. nationaler Handhabung wird dann gegebenenfalls noch ein Sicherheitszuschlag addiert. Die Wahl des tatsächlichen Freibordmaßes, welches die rechnerisch erforderliche Größe na- türlich überschreiten kann, obliegt dem Bauherrn bzw. seinem Planer. Je größer der Freibord ist, umso höher ist die systemimmanente Sicherheit des Bauwerks auch in Situationen, welche über die allgemeinen Bemessungsannahmen hinausgehen (Abb. 2). Ebenso lassen sich Änderungen der hydrologischen Grundlagen (z.B. der Bemessungshochwasserabflüsse) bei ausreichenden Freibordreserven in der Regel gut aufnehmen.

- Die Breite der Krone des Absperrbauwerks geht natürlich unmittelbar in die erforderliche Kubatur und den Raumbedarf ein. Bemessungsregeln konzentrieren sich oft auf eine ausreichende und sichere Befahrbarkeit des Bauwerks. Mit Blick auf später erforderliche - zum Zeitpunkt des Baus jedoch noch nicht bekannte - Anpassungsmaßnahmen an dem Absperrbauwerk der Talsperre ist eine über das unmittelbar erforderliche Maß hinausgehende Kronenbreite sehr günstig (Abb. 2). Breite Kronen erlauben $\mathrm{zu}$ gegebener Zeit die Durchführung von Baumaßnah- 

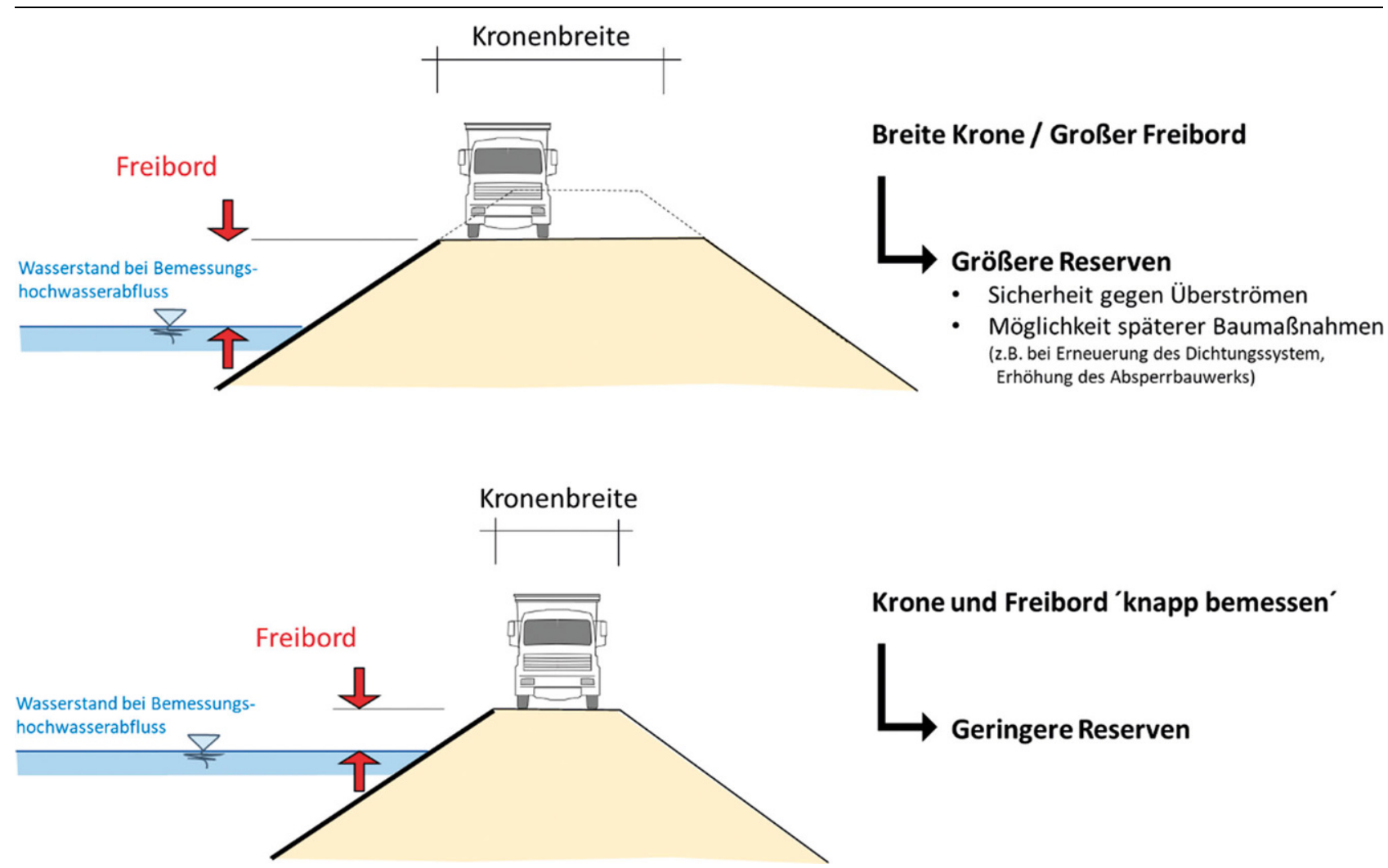

Abb. 2 Freibord und Kronenbreite

men (z. B. nachträglicher Einbau einer Dichtwand (Lang und Overhoff 2016), nachträglicher Einbau einer Oberflächendichtung, Injektionen im Untergrund, Erhöhung des Absperrbauwerks) und verbessern auch die systemimmanente Sicherheit in Situationen, welche über die Bemessung hinausgehen.

\section{Die Überwachung von Talsperren}

\subsection{Grundlagen}

Talsperren müssen überwacht werden. Der Umfang der Mitwirkung staatlicher Institutionen in der Talsperrenüberwachung ist in den einzelnen Ländern sehr unterschiedlich geregelt. Ebenso variieren die Existenz und die Bedeutung nationaler Regelwerke und nationaler technischer Empfehlungen in hohem Maße. Die Bandbreite reicht hier über die individuelle und sehr „persönliche“ Einbindung eines Expertengremiums in Österreich („Staubeckenkommission“), über ein vergleichsweise umfassendes nationales Regelwerk für Stauanlagen in Deutschland (DIN 19700, weitere technische Merkblätter (u.a. DWA)) bis hin zu einer Vielzahl von Ländern oh- ne explizite Regelungen und direkte technische Vorgaben für den Bau und Betrieb von Talsperren.

Was jedoch immer verbleibt, das ist die Verantwortung derjenigen, welche die Talsperren errichten bzw. betreiben. Diese direkte und unmittelbare Verantwortung ist in hohem Maße relevant. Es ist wichtig, dass beim Verkauf und bei der Übergabe von Stauanlagen dieser Aspekt den wirtschaftlich Verantwortlichen sehr deutlich kommuniziert wird. Auch eine scheinbar "nachrangige" Talsperre, welche im Rahmen eines Deals zwischen Großunternehmen als Bestandteil eines Kraftwerkssystems in ein neues organisatorisches Umfeld verschoben wird, ist und bleibt eine Stauanlage, welche ohne Unterbrechung und ohne Abstriche nach den allgemein anerkannten Regeln der Technik zu überwachen ist. Es hängt nun in hohem Maße von der Struktur der unmittelbaren Anlagenbetreuung ab, wie gut diese Übergänge, aber auch andere Wechsel in der Organisationsform vonstatten gehen.

Das Betreuungsverhältnis, d.h. das Verhältnis der Anzahl der zuständigen und fachkundigen Technikern und Technikerinnen bzw. Ingenieu- ren und Ingenieurinnen zur Anzahl der Talsperren, ist in den einzelnen Ländern und Regionen, aber auch bei den verschiedenen Betreibern in hohem Maße unterschiedlich. Aufgrund des dem wirtschaftlichen Leben immanenten Strebens nach immer weiteren Kostenreduzierungen zeigt sich vielerorts insbesondere bei den privatwirtschaftlich organisierten Betreibern eine deutlich erkennbare Verschlechterung dieses Betreuungsverhältnisses. In Zeiten schwieriger Randbedingungen, wie zum Beispiel aufgrund des wirtschaftlichen Drucks infolge sehr niedriger Energiepreise, verstärken sich diese Tendenzen. Damit einhergehend ist auch zu beobachten, dass immer mehr Aufgaben der Talsperrenbetreuung an Dritte vergeben werden. Grundsätzlich kann eine Veränderung der personellen Situation an einer Talsperre durchaus auch sehr positive Entwicklungen mit sich bringen. So erkennen Menschen, welche die Anlage bisher nicht kannten, möglicherweise mit neuer Aufmerksamkeit und anderem fachlichen Hintergrund andere Zusammenhänge als jene, welche seit sehr langer Zeit für die lokale Betreuung und Überwachung verantwortlich zeichnen. Nichtsdesto- 


\begin{tabular}{|c|c|c|c|c|c|}
\hline Aufgabe & Personenkreis & Verfügbarkeit & Betreuungsintensität & Betreuungsverhältnis & Anmerkung \\
\hline $\begin{array}{l}\text { Lokale Betreuung der } \\
\text { Anlage, Bauwerks- } \\
\text { überwachung }\end{array}$ & $\begin{array}{l}\text { Technisch ausgebildete } \\
\text { Personen mit detaillier- } \\
\text { ten Kenntnissen der } \\
\text { Talsperre }\end{array}$ & $\begin{array}{l}\text { Räumlich und } \\
\text { zeitlich hohe Ver- } \\
\text { fügbarkeit, kurze } \\
\text { Wege, Bereit- } \\
\text { schaftsdienst }\end{array}$ & Täglich bis wöchentlich & $\begin{array}{l}\text { Typischerweise eine bis meh- } \\
\text { rere Personen je Talsperre, } \\
\text { Sicherstellung einer aus- } \\
\text { reichenden Betreuung aller } \\
\text { Talsperren in besonderen Si- } \\
\text { tuationen (z. B. großräumiges } \\
\text { Hochwasserereignis) }\end{array}$ & $\begin{array}{l}\text { Typischerweise Ange- } \\
\text { stellte bzw. Angehörige } \\
\text { des Betreibers }\end{array}$ \\
\hline $\begin{array}{l}\text { Technische Ver- } \\
\text { antwortung für die } \\
\text { Talsperre }\end{array}$ & $\begin{array}{l}\text { Fachkundige(r) Talsper- } \\
\text { reningenieur(in) }\end{array}$ & $\begin{array}{l}\text { Gute Verfügbar- } \\
\text { keit, angemessene } \\
\text { Weglängen, Bereit- } \\
\text { schaftsdienst }\end{array}$ & $\begin{array}{l}\text { Nach Erfordernis, mindes- } \\
\text { tens wenige bis mehrere } \\
\text { Male jährlich }\end{array}$ & $\begin{array}{l}\text { Typischerweise eine bis meh- } \\
\text { rere Talsperren je Person, } \\
\text { Sicherstellung einer aus- } \\
\text { reichenden Betreuung aller } \\
\text { Talsperren in besonderen Si- } \\
\text { tuationen (z. B. großräumiges } \\
\text { Hochwasserereignis) }\end{array}$ & $\begin{array}{l}\text { Typischerweise Ange- } \\
\text { stellte bzw. Angehörige } \\
\text { des Betreibers, bei klei- } \\
\text { neren Anlagen ggfs. } \\
\text { langfristig an Dritte } \\
\text { ausgelagert }\end{array}$ \\
\hline $\begin{array}{l}\text { Technische Überprü- } \\
\text { fungen der Talsper- } \\
\text { ren }\end{array}$ & $\begin{array}{l}\text { Experten und Expertin- } \\
\text { nen aus den relevanten } \\
\text { Fachbereichen (u.a. } \\
\text { Wasserbau, Statik, } \\
\text { Geotechnik, Stahlwas- } \\
\text { serbau) }\end{array}$ & $\begin{array}{l}\text { Im Rahmen der } \\
\text { Aufträge des } \\
\text { Betreibers }\end{array}$ & $\begin{array}{l}\text { Im Zuge der in größeren } \\
\text { zeitlichen Abständen (meh- } \\
\text { rere Jahre) erforderlichen } \\
\text { technischen Überprüfungen }\end{array}$ & - & $\begin{array}{l}\text { Externe Fachleute bzw. } \\
\text { Fachbüros im Auftrag } \\
\text { des Betreibers }\end{array}$ \\
\hline
\end{tabular}

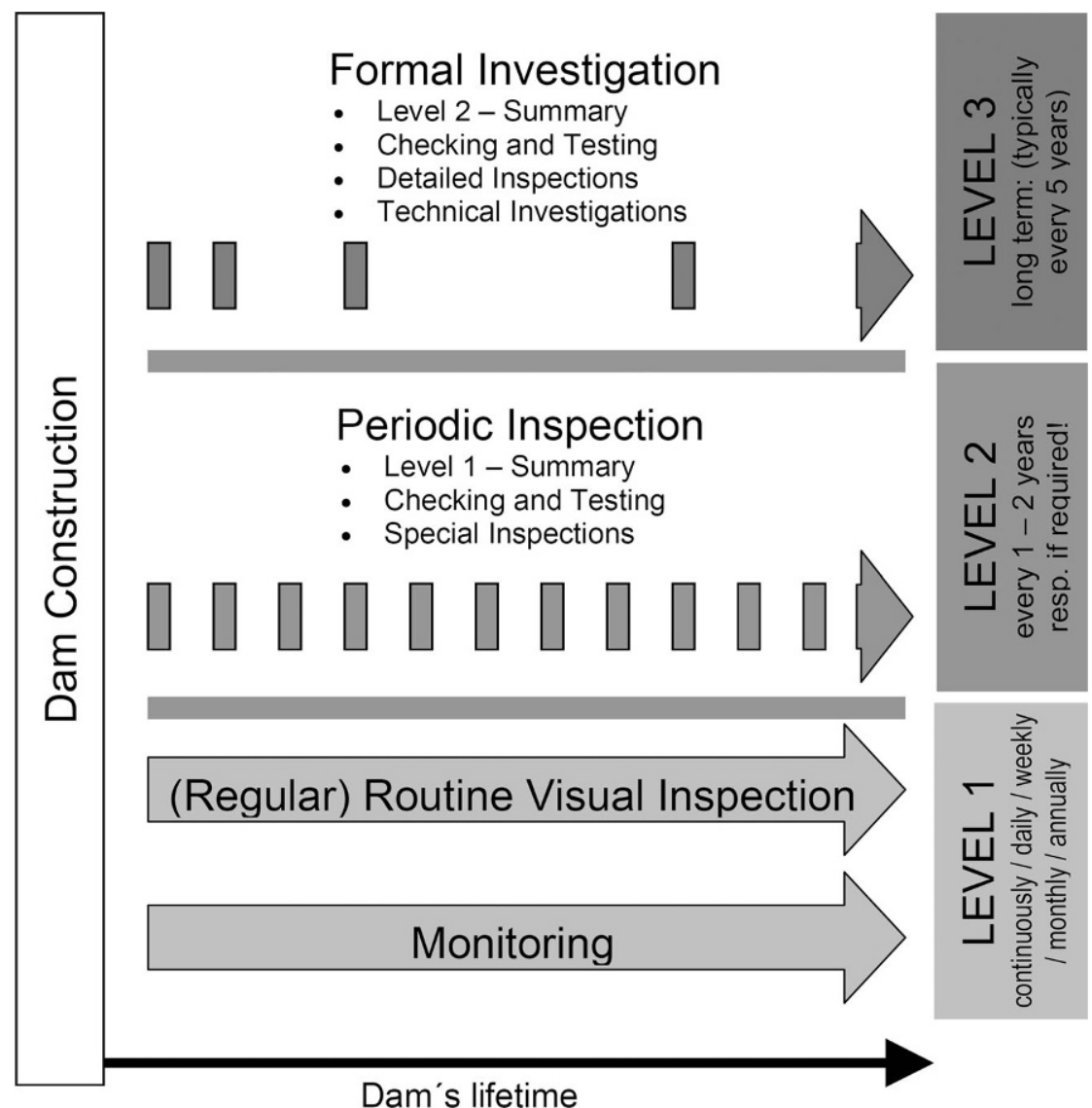

Abb. 3 Darstellung der Aktivitäten bei der Talsperrenüberwachung während der Lebenszeit einer Talsperre (Abb. aus dem ICOLD Bulletin 158; ICOLD 2014) trotz ist die lokale Expertise und die detaillierte Kenntnis der spezifischen Talsperre unabdingbare Voraussetzung für eine gute und zuverlässige technische Betreuung einschließlich der Bauwerksüberwachung. Aus Sicht des Verfassers sind bei der Organisation der Überwachung von Talsperren folgende Grundsätze hinsichtlich der erforderlichen Personalressourcen aus Sicht des Betreibers einzuhalten (siehe Tab. 1).

Bei bestehenden Anlagen gilt es nun, sich insbesondere im Zuge der Talsperrenüberwachung immer wieder - und auch immer wieder ,von Neuem“ (!) - mit allen sicherheitsrelevanten Anlagenteilen zu beschäftigen. Die Überwachung des Zustandes des Absperrbauwerks und aller anderen sicherheitsrelevanten Anlagenteile spielt hier eine herausragende Rolle. Von Zeit $\mathrm{zu}$ Zeit sind aber auch die anderen Aspekte der Talsperrensicherheit einschließlich der ursprünglichen Dimensionierung (u.a. Hydrologie, Hydraulik der Hochwasserentlastungsanlage, Lastannahmen, Konstruktionsprinzip, Tragsicherheitsnachweise - siehe auch Kap. 1.) einer grundlegenden Überprüfung zu unterziehen. Nur so kann die Sicherheit von Talsperren über viele Jahrzehnte bzw. über Jahrhunderte (!) in die Hände der nachkommenden Generationen gelegt werden.

Es ist daher zwingend notwendig, die Überwachung von Talsperren auf unterschiedlichen zeitlichen und fachlichen Ebenen durchzuführen. Eine Qualitätssicherung dieser Arbeiten ist dringend anzuraten. Verantwortlich für 


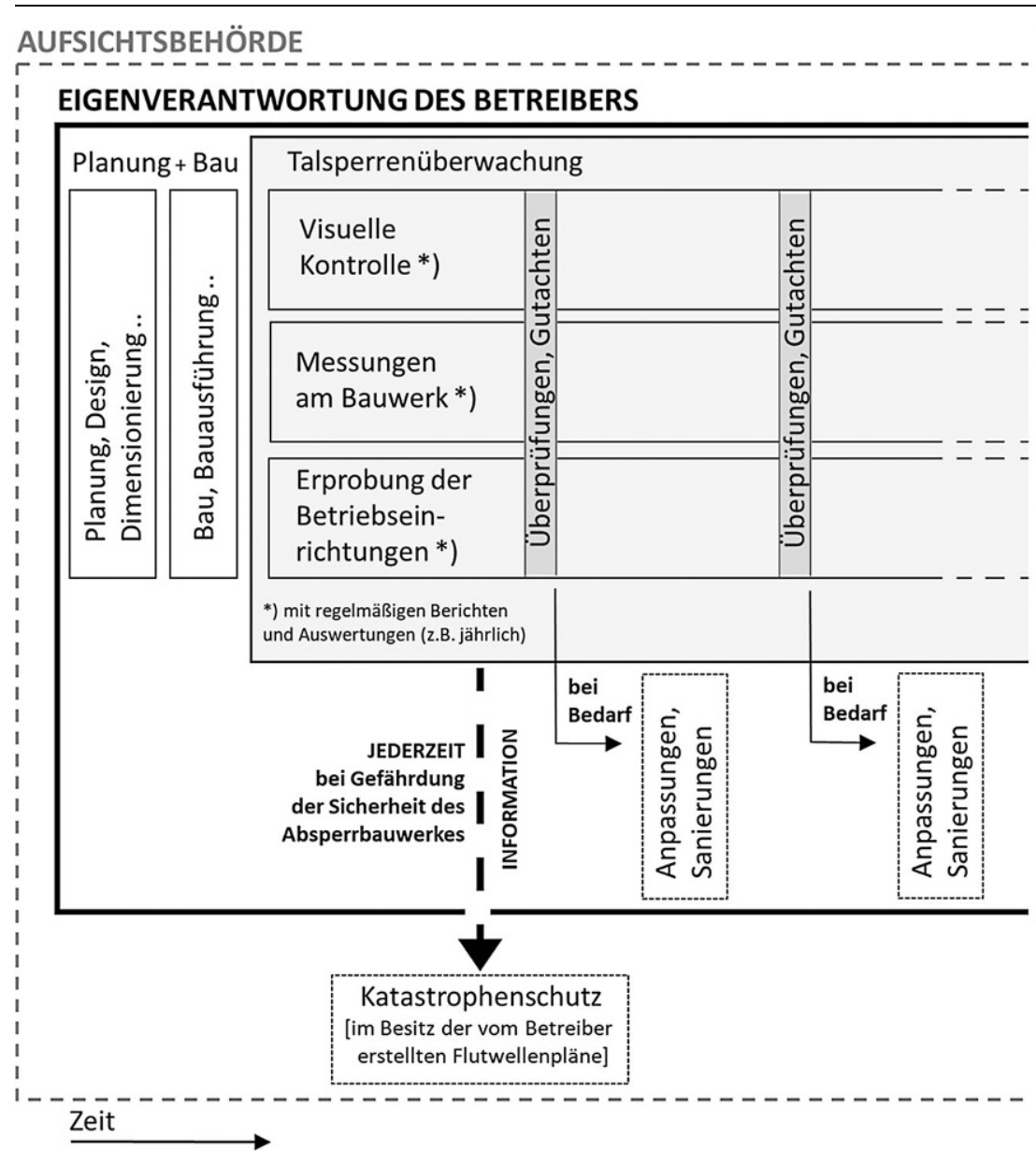

Abb. 4 DieÜberwachung von Talsperren(allgemeine Darstellung ohne direkten Bezug auf nationale Regelwerke)

alle Maßnahmen der Talsperrenüberwachung ist - weitgehend unabhängig von den nationalen Regelwerken und Empfehlungen - der Betreiber. Im Rahmen der Gremienarbeit der International Commission on Large Dams (ICOLD) wurden allgemeine Empfehlungen zur Überwachung von Talsperren erarbeitet (ICOLD 2009, 2014). Hier wird die Überwachung von Talsperren auf verschiedenen Niveaus, d.h. mit unterschiedlicher fachlicher Tiefe und in variierenden zeitlichen Intervallen, beschrieben (Abb. 3). In dieser Empfehlung werden für die unterschiedlichen Ebenen bzw. Niveaus (vgl. „Level“ (engl.) in Abb. 3) der Talsperrenüberwachung Größenordnungen der zeitlichen Abfolge vorgeschlagen. Diese sollen unter anderem die Größe und das Gefährdungspotenzial der jeweiligen Talsperre berücksichtigen.

\subsection{Konzept zur Talsperrenüberwachung}

Im Sinne einer allgemeinen Darstellung, welche sich in wichtigen Aspekten auf Vorgaben in verschiedenen internationalen, nationalen und regionalen Regelwerken bzw. Empfehlungen bezieht, wird nachfolgend ein grundlegendes Konzept für die Qualitätssicherung und die Überwachung von Talsperren skizziert (Abb. 4):

- Die Eigenverantwortung des Betreibers muss der Kern der Talsperrenüberwachung sein. Die Rolle und die Intensität der Mitwirkung der Aufsichtsbehörde hängen in hohem Maße von den nationalen und regionalen Festlegungen bzw. der jeweils gelebten Praxis ab. Ebenso sollten die Größe bzw. die Gefährdungspotenziale der Talsperren Einfluss auf den Umfang der Mitwirkung der Aufsichtsbehörde an der Talsperrenüberwachung haben. Aus Sicht des Autors ist es jedoch zwingend, dass trotz etwaiger staatlicher Mitwirkung die grundsätzliche Verantwortung deutlich sichtbar bei den Betreibern verbleibt. Diese sind dafür zuständig, dass ihre Anlagen den allgemein anerkannten Regeln der Technik entsprechen. Als ,anerkannte Regel der Technik" wird eine Festlegung verstanden, die von einer Mehrheit repräsentativer Fachleute als Wiedergabe des Stands der Technik angesehen wird (siehe auch Europäische Norm EN 45020).

- Selbstverständlich liegt es auch in der Verantwortung des Betreibers bzw. des Unternehmens oder der Institution, welche die Planung und den Bau der Talsperre initiiert und realisiert, dass die Planung, das Design, die Dimensionierung, aber auch der Bau und die Bauausführung den hohen Ansprüchen genügen, welche sich aus der Bedeutung der Talsperren ableiten lassen. Hierbei sollte bereits dem potenziellen Gefährdungspotenzial (auch im Sinne einer langfristigen Prognose der räumlichen Entwicklung) und der gleichsam unbegrenzten Standzeit dieser Anlagen Rechnung getragen werden.

- Die Talsperrenüberwachung beginnt bereits in der Bauphase und dauert im Sinne einer generationsübergreifenden Aufgabe unbegrenzt an. Nur im Falle eines Rückbaus einer Talsperre würde sie entfallen. Das grundlegende und kontinuierlich umzusetzende „Basismodul“ der Talsperrenüberwachung umfasst drei wesentliche Aktivitäten (siehe auch Abb. 4, grau hinterlegter Bereich):

- Die Visuelle Kontrolle ist ein zentrales und unersetzliches Element der Bauwerksüberwachung an kleinen und großen Talsperren. Sie ermöglicht eine umfassende qualitative Beurteilung des Bauwerkszustandes und dessen unmittelbarer Umgebung. Es ist zwingend erforderlich, dass die visuelle Kontrolle von geschultem Personal durchgeführt wird, welches mit der Anlage gut vertraut ist (siehe auch Tab. 1). Im Rahmen von visuellen Kontrollen wird erfahrungsgemäß der größte Teil der Mängel und Schäden an einer Talsperre erkannt. Visuelle Kontrollen sollten unter Beachtung der Größe und des Gefährdungspotenzials einer Talsperre im zeitlichen Abstand von einem Tag bis sehr weni- 
gen Wochen durchgeführt werden. Technische Empfehlungen (z.B. DWA 2011) geben in exemplarischer Weise Hinweise zum Umfang und Vorgehen der visuellen Kontrolle.

- Neben der Messung des Zu- und Abflusses sowie einer zwingenden Möglichkeit der Bestimmung des Wasserstands im Stauraum werden weitere Messungen am Bauwerk durchgeführt. Diese variieren hinsichtlich ihrer Art und Weise und ihres Umfangs in erheblichem Maße. Ziel der Messungen ist es, den Zustand des Absperrbauwerks und anderer wichtiger Anlagenteile zu erfassen. Neben Deformationsmessungen werden insbesondere auch Messungen zur Beobachtung der Durchsickerung durchgeführt. Eine zentrale Bedeutung kommt der messtechnischen Bestimmung des Sickerwasserabflusses zu. Die Messungen werden mit unterschiedlicher Häufigkeit (z. B. permanent, täglich, wöchentlich etc.) vorgenommen. Wichtige Messdaten (z. B. Wasserstand und Sickerwasserabfluss) sollten nach Möglichkeit automatisch erfasst und fernübertragen werden.

- In regelmäßigen Abständen (z. B. 6 Monate, 12 Monate) ist eine $\mathrm{Er}$ probung der Betriebseinrichtungen erforderlich. Hierbei sollten insbesondere die sicherheitsrelevanten beweglichen Anlagenteile, d. h. der Grundablass und etwaige Verschlüsse an der Hochwasserentlastungsanlage, auf ihre Einsatzbereitschaft und Funktionalität hin überprüft werden.

Die Ergebnisse dieser Aktivitäten sollten im Sinne des kontinuierlichen Ablaufs des Basismoduls der Talsperrenüberwachung in regelmäßigen Abständen zusammengefasst werden. Hierbei sind die Ergebnisse der visuellen Kontrolle und die Daten der Bauwerksüberwachung darzustellen und zu bewerten.

- In größeren Zeitabständen (z. B. 5 Jahre, 10 Jahre, 15 Jahre) ist eine eingehende Überprüfung („Formal Investigation“, „Vertiefte Überprüfung“) der Talsperre durchzuführen. Es gilt hierbei, die Anlage ohne Vorbehalte und frei von Vorprägungen auf ihre Sicherheit hin $\mathrm{zu}$ überprüfen (siehe auch Abschn. 2.1.). Im Laufe der Zeit werden sich früher oder später an jeder Talsperre Erfordernisse zur
Anpassung und Sanierung der Anlage ergeben. Typischerweise werden diese im Rahmen dieser Überprüfungen festgestellt und im Anschluss innerhalb eines angemessenen Zeitraumes umgesetzt. Dieser Prozess ist von wesentlicher Bedeutung, um die Sicherheit der Talsperre über Generationen hinweg unter Beachtung der jeweils gültigen gesellschaftlichen Sicherheitsansprüche bzw. dem jeweils gültigen Stand der Technik entsprechend zu gewährleisten.

- $\mathrm{Zu}$ jeder Zeit muss eine Gefährdung der Standsicherheit des Absperrbauwerks oder anderer sicherheitsrelevanter Anlagenteile erkannt werden können. Auch dies liegt zwingend in der Eigenverantwortung des Betreibers. Es muss gewährleistet sein, dass in diesem Fall eine qualifizierte und möglichst rasche Information an die jeweils zuständige Behörde oder Institution (z.B. den Katastrophenschutz) ergeht. In vielen Ländern ist die Ausarbeitung von Bruchszenarien und die Weitergabe entsprechender Planunterlagen an die zuständigen öffentlichen Stellen inzwischen Realität oder zumindest in die Wege geleitet worden. Die Durchführung von Evakuierungen und alle Maßnahmen des Katastrophenschutzes obliegen im Sinne der Mächtigkeit und Besonderheit dieser Aufgabe nun nicht mehr dem Betreiber, welchem in aller Regel die Strukturen und Kompetenzen hierfür fehlen. Dies entbindet ihn allerdings in keiner Weise von seiner Verantwortung.

\section{Beiträge zu verschiedenen Aspekten der Sicherheit von Talsperren}

\section{1 Überblick}

Nachfolgend werden verschiedene Aspekte der Sicherheit von Talsperren im besonderen Kontext zu aktuellen Entwicklungen und Konzeptionen vorgestellt und diskutiert. Von besonderer Bedeutung ist hierbei jeweils der Blick auf die „unbeschränkten“ Standzeiten bzw. Lebensdauern der Talsperren und die generationsübergreifende Aufgabe der Talsperrenüberwachung.

\subsection{Die Bedeutung von Grundablässen für die Talsperrensicherheit}

Grundablässe besitzen unterschiedliche Zielsetzungen. Diese können von der Wasserspiegelabsenkung in Notsituationen, über die Entleerung im Rahmen von Sanierungen, der Abgabe von Restwasser, der Mitwirkung bei der Hochwasserabfuhr bis hin zum Sedimentmanagement reichen. Weltweit gesehen variiert die Handhabung der Bemessungsansätze für Grundablässe sehr stark. In vielen Ländern sind Grundablässe unabdingbarer Bestandteil der Sicherheitsphilosophie. Es gibt weltweit jedoch sehr viele Talsperren, welche über keinen Grundablass verfügen. Bei anderen Anlagen existieren zwar Grundablässe, jedoch sind diese aufgrund fehlender Instandhaltung und Sedimentablagerungen oft nicht mehr funktionsfähig (Aufleger 2013).

Im Sinne der Notwendigkeit, Talsperren von einer Generation zur nächsten $\mathrm{zu}$ übergeben, sind Grundablässe an Talsperren als in hohem Maße relevante Einrichtungen $\mathrm{zu}$ sehen. Sie können insbesondere in Notsituationen von größtem Nutzen sein und geben grundsätzlich eine große Flexibilität in unterschiedlichsten Betriebsfällen. Fallbeispiele ohne Grundablass können nicht als Begründung für den Verzicht auf entsprechende Auslässe an neuen Talsperren dienen. Dies gilt auch für Anlagen im Ausland. Für den langfristigen Betrieb von Grundablässen ist es zwingend notwendig, mindestens zwei voneinander unabhängig bedienbare Verschlüsse anzuordnen (vgl. Abb. 5). Diese Forderung findet sich auch in verschiedenen nationalen Regelwerken (u.a. DIN 19700). Die Unabhängigkeit der beiden Verschlüsse ist die Voraussetzung für eine betriebssichere regelmäßige Erprobung des Grundablasses (Abb. 5). Das Risiko des Verbleibs ungenutzter Stahlwasserbauteile bei vergleichsweise großen Druckhöhen (Fallbeispiel in Abb. 6) kann damit so gut wie ausgeschlossen werden. Die langfristige Betriebssicherheit von Grundablässen kann allerdings nur bei einem von Anfang an nachhaltigen Sedimentmanagement gewährleistet werden. Auch dies ist eine Aufgabe, welche mit großer Weitsicht wahrgenommen werden muss.

\subsection{Bauwerksüberwachung mit Generationen von Messsystemen und Messverfahren}

In der Bauwerksüberwachung kommen die unterschiedlichsten Verfahren und Messgeräte zum Einsatz. Die hierbei verwendeten Technologien richten sich 

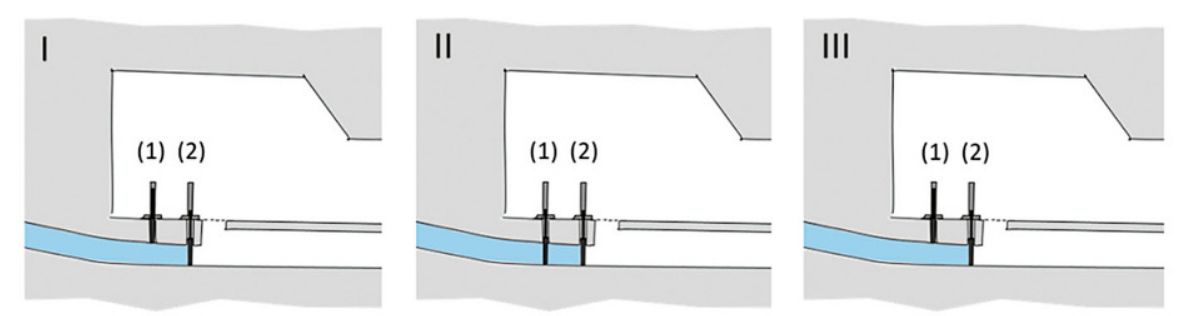

\author{
Möglichkeit der Nass- \\ Erprobung von Verschluss 1 \\ (ohne Druckdifferenz)
}

(1) (2)
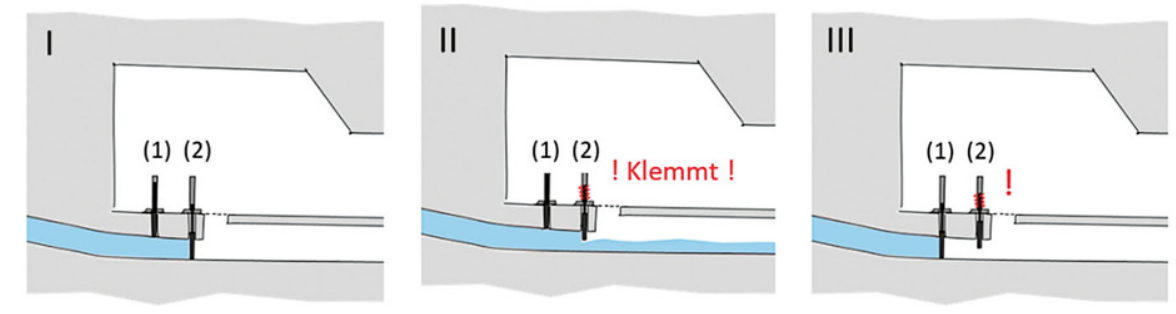
Betriebssicherheit bei Erprobung [Beispiel]
Klemmen von Verschluss 2 im Zuge einer Erprobung $\rightarrow$
Stopp des Volumenstroms durch Verschluss 1

Abb. 5 Grundablass mitzwei Verschlüssen $\rightarrow$ gute Voraussetzung für den Erhalt der Betriebssicherheitüber Generationen (hier Beispiele der hohen Flexibilität bei der Grundablassüberprüfung bei Vorhandensein von zwei Verschlüssen)
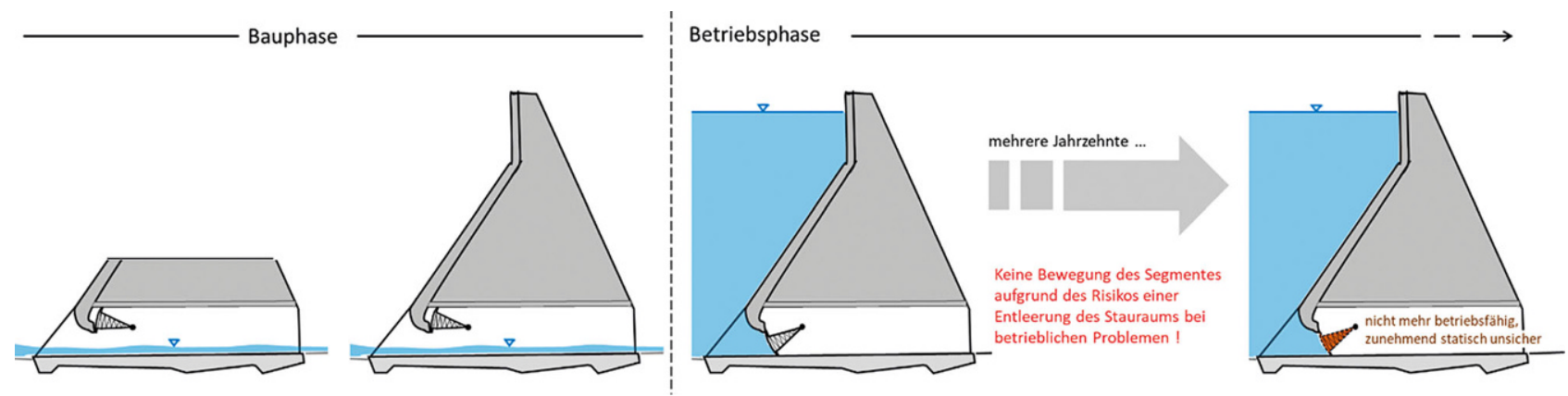

Abb. 6 Grundablass mit einem Verschluss $\rightarrow$ großes Risiko einer abnehmenden Betriebssicherheit und eines sich langsam entwickelnden Sicherheitsproblems im Laufe der Betriebszeit (Fallbeispiel)

zunächst nach dem Stand der entsprechenden Messtechnik zum Zeitpunkt des Baus der Talsperre. Bei größeren Anlagen wurden auch verschiedenste Messgeräte zum Beispiel zur Bestimmung von Verformungen, Drücken und Temperaturen in das Bauwerk in einer Art und Weise integriert, welche eine spätere Auswechslung oder eine Reparatur ausschließt. Bezogen auf die „unbegrenzte Lebensdauer" der Absperrbauwerke muss davon ausgegangen werden, dass diese Messgeräte im Laufe der Jahre oder Jahrzehnte in ihrer Zuverlässigkeit und Funktionalität deutliche Einschränkungen erleiden und defekt werden. Im Laufe dieser Zeit können jedoch möglicherweise sehr wichtige und umfassende Daten über das Bauwerksverhalten gewonnen werden. Dennoch muss beachtet werden, dass spätestens nach mehreren Jahrzehnten die Lebensdauer der meisten innerhalb der Absperrbauwerke integrierten Messgeräte erreicht wird. Diese Situation stellt die Verantwortlichen vor nicht unerhebliche Probleme. Es gilt $\mathrm{zu}$ entscheiden, ob ein Ersatz der Messgeräte technisch möglich und sinnvoll ist, ob die erforderlichen Messgrößen (Bauwerksreaktion) möglicherweise über andere Verfahren in besserer Art und Weise ermittelt werden können oder ob der Wegfall der bisherigen Informationen aus dem Bauwerk akzeptiert werden kann.

Einen besonderen Stellenwert nehmen hierbei die Messgeräte ein, welche im Inneren von Staudämmen installiert werden. Staudämme unterscheiden sich von Staumauern insbesondere durch ihre erheblich geringeren Anforderungen an die Tragfähigkeit des Untergrundes und einer wesentlich größeren Flexibilität hinsichtlich der Bauformen und Ausgestaltungen. Hinsichtlich der geringen Widerstandsfähigkeit in Überströmungssituationen und der potenziellen Versagensform der inneren Erosion bedingen sie jedoch oft einen bedeutsamen Aufwand in der Bauwerksüberwachung. Aus Sicht des Verfassers sind beim langfristigen Umgang mit Messgeräten in Staudämmen folgende Aspekte von besonderer Bedeutung:

- Messgeräte, die im Inneren eines Staudammes installiert werden, können insbesondere im Bereich eines natürlichen Kerns potenzielle Schwachstellen oder lokale Störungen verursachen. Grundsätzlich bieten Kabel und Schläuche, welche $\mathrm{zu}$ den einzelnen Messgebern führen, günstige Randbedingungen für Sickerströmungen. Im Bereich von Messgeräten sind die Möglichkeiten der Verdichtung sowie der Verdichtungskontrolle mitunter nicht unerheblich beeinträchtigt. Im Zuge der Planung der ersten Messausstattung eines Staudamms sollten daher der 


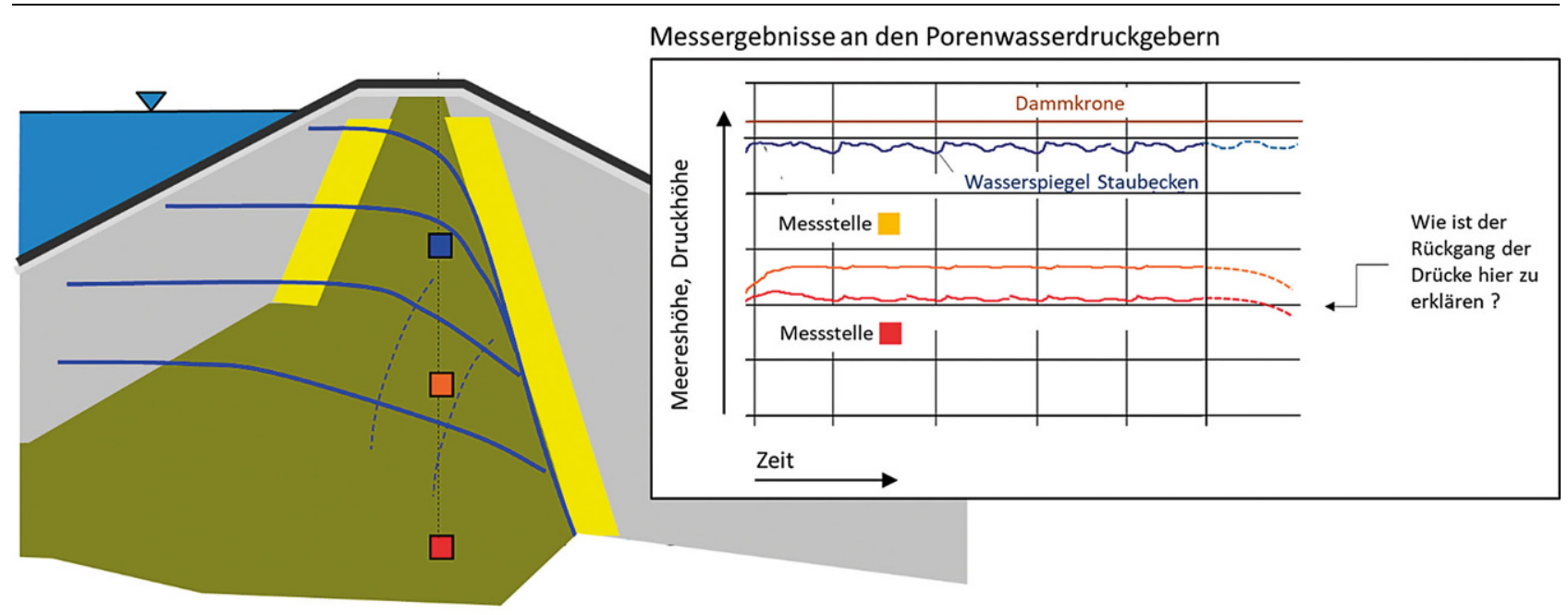

\section{Interpretation A}

Zunehmende Dichtung im Stauraum aufgrund Ablagerungen von Feinteilen (unkritisch!)

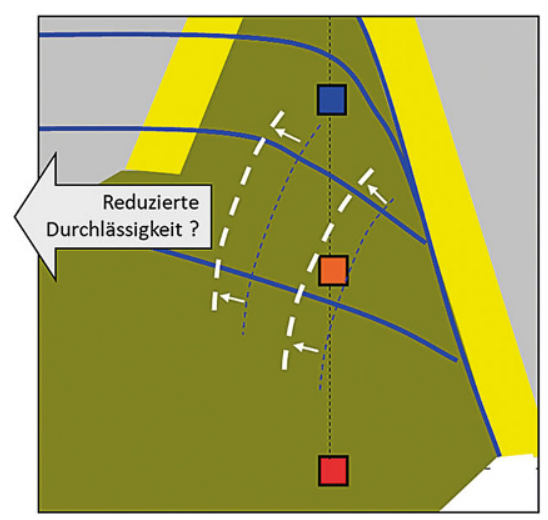

\section{Interpretation B}

Zunehmende Durchlässigkeit im Kern unterwasserseitig der Messstellen (möglicherweise kritisch, innere Erosion ?)

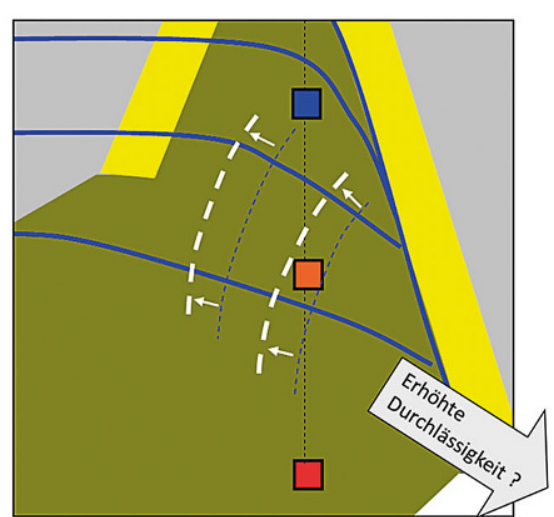

\section{Interpretation C}

Messfehler, Probleme am Gesamtsystem (schwierig zu definieren, ggfs. unkritisch)

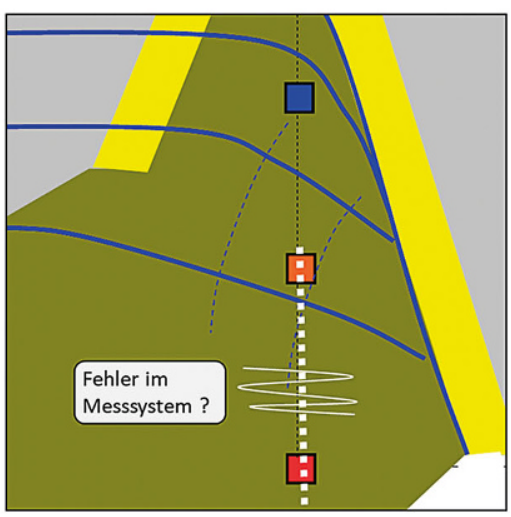

Abb. 7 Mögliche Entwicklung der Porenwasserdrücke im Kern eines Staudammes und eine Auswahl an Interpretationsmöglichkeiten (fiktives Fallbeispiel)

Umfang, die Anzahl und insbesondere auch die Anordnung der Messleitungen kritisch geprüft werden. Insbesondere in natürlichen Kernen ist daher grundsätzlich eine sehr deutliche Zurückhaltung hinsichtlich der $\mathrm{zu}$ installierenden Messausstattung zu empfehlen.

- Der Einsatz von Messgeräten zur Ermittlung von Totalspannungen (Erddruckgeber) sollte in jedem Einzelfall grundsätzlich auf seine Sinnhaftigkeit überprüft werden. Von besonderer Bedeutung sind hierbei die erheblichen Unsicherheiten hinsichtlich der Qualität der Messergebnisse bzw. das grundsätzliche Erfordernis der nachträglichen Bearbeitung bzw. Kalibrierung der Rohmessdaten anhand der anfänglichen Messwertentwicklung (Aufleger 2007).

- Porenwasserdruckmessungen können Messdaten von besonderer Bedeutung beispielsweise über den Druckabbau in einem natürlichen Kern liefern. Für die Interpretation der Entwicklung der Messdaten ist allerdings umfassende Erfahrung erforderlich. So können ausgeprägte Messwertentwicklungen durchaus unterschiedliche Ursachen haben. Fallweise ist ein nicht unerheblicher Interpretationsspielraum gegeben (Abb. 7).

- Die Nachrüstung von Messgeräten muss mit besonderer Sorgfalt geplant bzw. in jedem Fall auf ihre Sinnhaftigkeit überprüft werden. Insbesondere ist beim nachträglichen Einbau von Messgebern mittels Bohrungen in natürlichen Kernen und im Untergrund von Staudämmen besondere Sorgfalt geboten. Das Bohren mit Wasser oder Luft muss so weit wie möglich vermieden werden. Wenn es keinen anderen Ausweg gibt, muss es mit größter Sorgfalt und Überwachung durchgeführt werden, um das Risiko von hydraulic fracturing oder pneumatic fracturing durch die Begrenzung der Luft-und Wasserdrücke zu reduzieren (ICOLD 2014). Die jeweils zulässigen Werte hängen von der Materialdichte, dem Porenwasserdruck und der Tiefe des Bohrlochs ab. Bei der Nachrüstung von Messgeräten in sensitiven Bereichen von Absperrbauwerken (insbesondere bei Staudämmen mit natürlichen 

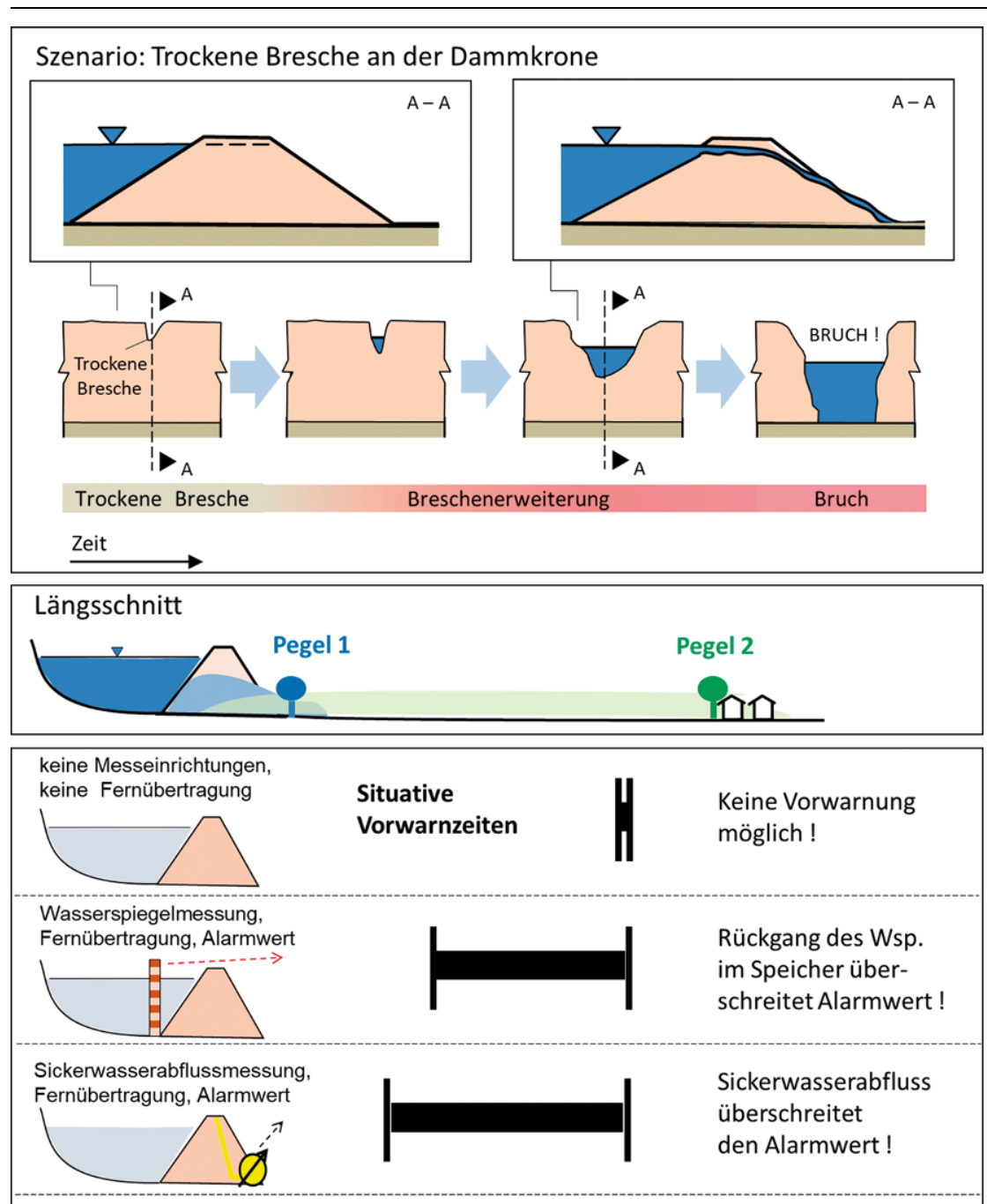

Abfluss, Wasserspiegel

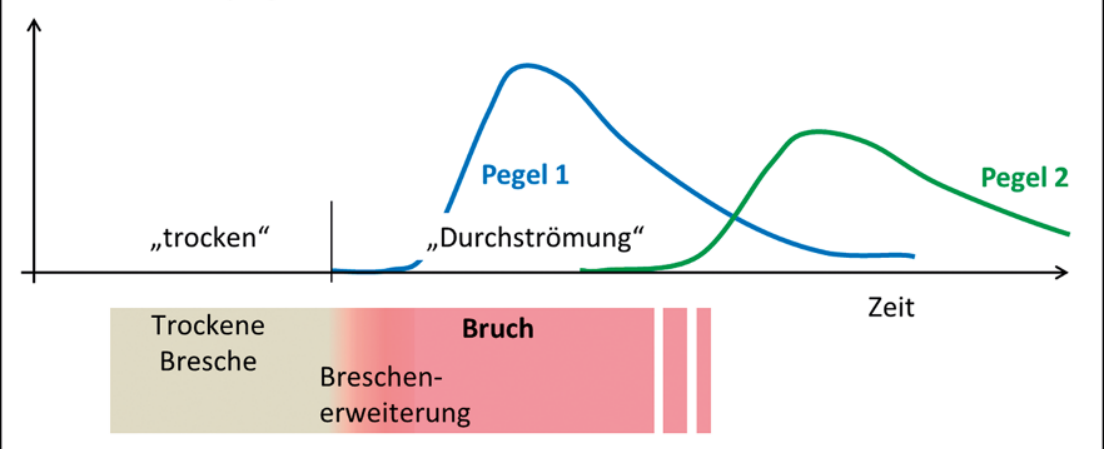

Abb. 8 Fallbeispiel: Trockene Bresche mit anschließenden Staudammbruch, Darstellung der erheblichen Schwankungsbreite der situativen Vorwarnzeiten in Abhängigkeit vom Umfang der Bauwerksmessungen

Kernen) sollten Techniken mit deutlich geringerem Risiko (u. a. Schnecken und sonic drilling) in Erwägung gezogen werden.

- Messmethoden und Messgeräte werden ständig weiterentwickelt. Moderne Messverfahren, welche berüh- rungslos funktionieren (z.B. Laserscan, Fotogrammetrie) besitzen ein sehr großes Potenzial und eröffnen neue Einblicke auf die Anlage bzw. auf einzelne Anlagenteile (z. B. Verformungsmessung, Rissdetektion, optische Kontrollen). Sie sind in ihrer
Wirksamkeit bzw. „Eindringetiefe“ jedoch auf die freien Oberflächen der Bauwerke begrenzt. Andere moderne Messverfahren sind in der Lage, entlang von sehr robusten und sehr langlebigen Glasfaserleitungen, welche ohne jede weitere Messgeräte in verschiedenen Bauwerksteile integriert werden können, wichtige Informationen über die Temperatur, über Sickerströmungen und auch in einzelnen Fällen über die Verformungen in verteilter Form zu ermitteln. Die eigentliche Messtechnik und damit jene Messkomponenten, welche einer relevanten Alterung, aber auch einer ständigen technischen Weiterentwicklung unterliegen, werden hierbei außerhalb des Bauwerkskörpers angeschlossen und sind somit jederzeit zugänglich und austauschbar. Solche Messverfahren (z. B. faseroptische Temperaturmessungen zur Leckageortung; Fry et al. 2007) bieten gegenüber klassischen Verfahren mitunter deutliche längerfristige Messoptionen und weisen meist eine sehr große Datendichte auf.

\subsection{Bruchszenarien und Vorwarnzeiten an Staudämmen - Sicherheit für Generationen}

Die Sicherheit der Talsperren ist elementar. Mithilfe von Flutwellenberechnungen werden die Auswirkungen des Versagens von Absperrbauwerken in hypothetischer Form ermittelt. Diese Untersuchungen sind Grundlage für vorbereitende Maßnahmen des Katastrophenschutzes. In diesen Szenarien wird davon ausgegangen, dass das Versagen der Staumauer oder des Staudamms bereits in einem sehr frühen Entwicklungsstadium erkannt wird. Die vorhandenen Methoden der Bauwerksüberwachung sind hierfür jedoch nur bedingt geeignet. Es liegt im Kern der Aufgabenstellung und der Sicherheitsanforderung unserer Gesellschaft, dass zu jedem Zeitpunkt ab der Fertigstellung einer Talsperre sichergestellt werden muss, dass ein Versagen der Stauanlage zu einem möglichst frühen Zeitpunkt erkannt werden muss. Ebenso ist sicherzustellen, dass schadhafte Einwirkungen auf Talsperren durch Dritte so rechtzeitig erkannt werden können, dass Gegenmaßnahmen möglich sind.

In den Modellvorstellungen wird bei Staudammbrüchen als mögliche Vorwarnzeit die Zeitdauer angegeben, wel- 


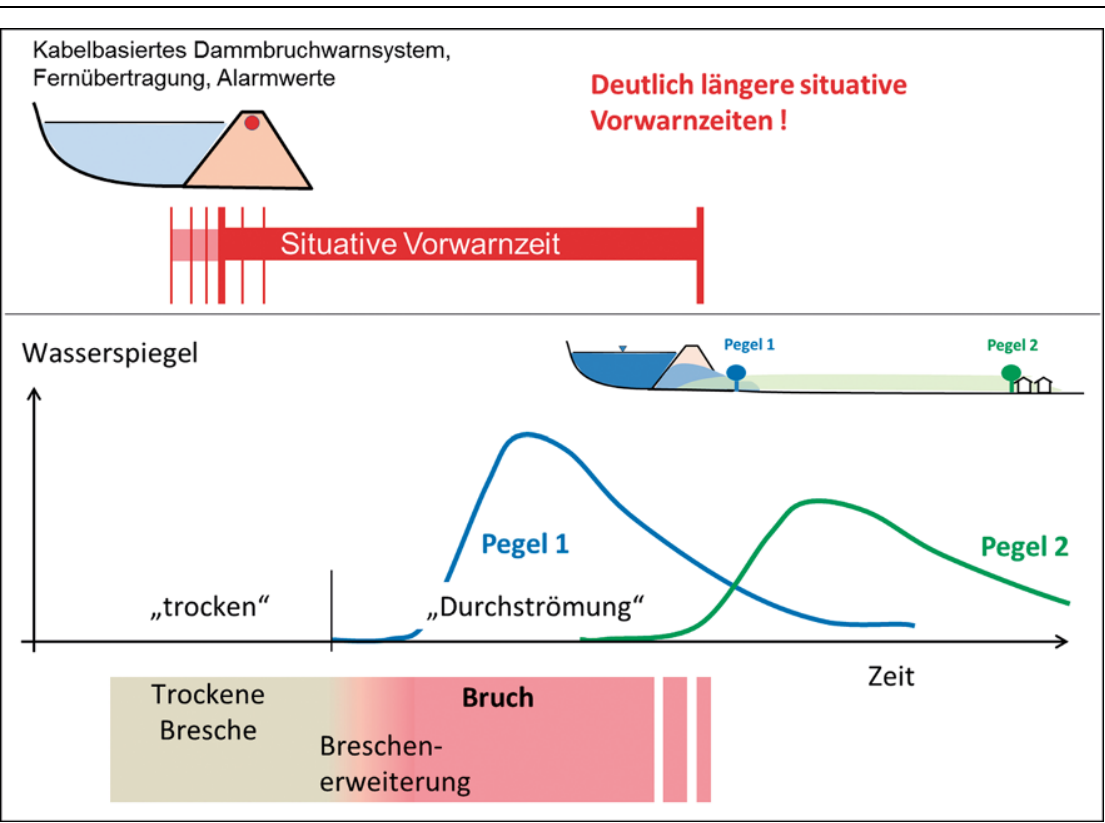

Abb. 9 Fallbeispiel: Trockene Bresche mit anschließenden Staudammbruch-Darstellung der deutlich längeren situativen Vorwarnzeit bei Einsatz eines kabelbasierten Dammbruchwarnsystems

che zwischen dem Beginn der Bildung einer Bresche im Dammkörper und dem Auftreten kritischer hydraulischer Zustände im betrachten Bereich im Unterwasser verstreicht. Hierbei wird implizit davon ausgegangen, dass die Breschenbildung unmittelbar erkannt wird und die Alarmmeldung unverzüglich erfolgt. Brüche von Dammbauwerken können durch unterschiedliche Prozesse ausgelöst werden. Analysiert man historische Dammbrüche, so ist die häufigste Ursache das Überströmen, gefolgt von innerer Erosion. Weitere Ursachen können im Untergrund oder auch im Aufbau des Dammkörpers liegen. Gemeinsam ist den verschiedenen Versagensprozessen, dass es fast immer früher oder später $\mathrm{zu}$ einer Situation kommt, in welcher die Dammkrone überströmt wird (Lopez und Aufleger 2017). Hieran schließt sich bei üblichen Dammkonstruktionen unvermeidbar die Bildung einer Bresche mit einem progressiven Anstieg der Flutwelle an. Diese kann die Größenordnung natürlicher Hochwasserereignisse um Größenordnungen übertreffen und katastrophale Auswirkungen haben.

Tatsächlich hängt die Wahrscheinlichkeit, dass das beginnende Versagen eines Staudammes bereits $\mathrm{zu}$ einem sehr frühen Zeitpunkt erkannt wird, insbesondere von der Art und dem Umfang der vorhandenen Messeinrichtung sowie den grundsätzlichen Umstän- den ab. Im Zuge großer Hochwasserereignisse ist die allgemeine und auch die anlagenspezifische Aufmerksamkeit deutlich erhöht. In diesen Fällen wird ein Überströmen der Dammkrone sehr frühzeitig erkannt werden. Bei anderen Schadensursachen - insbesondere bei sehr raschen Schadensentwicklungen unter „Schönwetterverhältnissen“ - ist davon auszugehen, dass eine Schadensentwicklung nur durch fernübertragene Messsignale oder zufällige Beobachtungen vor dem Eintreffen einer Flutwelle im Unterwasser erkannt werden kann.

Solche Situationen sind beispielsweise bei Prozessen der inneren Erosion (insbesondere bei fehlenden Sickerwasserabflussmessungen) oder aber auch bei trockenen Breschen im Bereich der Dammkrone vorstellbar. Unter trockener Bresche wird eine Schadeinwirkung verstanden, welche eine massive Veränderung der Dammkrone verursacht. Sobald die Unterkante dieser Bresche unter dem Wasserstand im Staubecken liegt, beginnt eine Durchströmung dieses Bereiches und in der Folge eine progressive Vergrößerung der Schadstelle. Je nach Umfang der messtechnischen Ausstattung des Staudammes variieren nun die „situativen Vorwarnzeiten" in erheblichem Maße (Abb. 8).

- Bei Anlagen ohne weitergehende messtechnische Ausstattung, d.h. insbesondere ohne die Möglichkeit der Fernübertragung von Messdaten, kann nicht davon ausgegangen werden, dass eine Vorwarnung der Bevölkerung unter diesen Umständen möglich ist. Das Entstehen der Bresche könnte außerhalb der visuellen Kontrollen nur durch zufällig Beobachtungen anderer Personen erkannt werden.

- Falls der Beckenwasserstand automatisch an eine zentrale Leitstelle übergeben wird oder beispielsweise über eine Alarmwertvorgabe mit einem Bereitschaftsdienst verbunden ist, kann das sich abzeichnende Versagen erkannt werden, sobald es zu einem erkennbaren und ungewöhnlichen Absinken des Wasserstandes im Staubecken kommt. Dies ist jedoch erst in einem sehr weit fortgeschrittenen Stadium der Breschenbildung der Fall.

- Bei Staudämmen, welche über eine gut funktionierende Sickerwasserabflussmessung verfügen, wird die beginnende Einströmung in die Bresche nach dem Auffüllen der entsprechenden Drainage- und Sickerzonen bzw. -leitungen zu einer signifikanten Erhöhung der Messwerte führen. Hierdurch kann das sich abzeichnende Problem an der Dammkrone möglicherweise noch vor dem Absinken des Wasserspiegels im Staubecken erkannt werden.

Ein unmittelbares Erkennen der Schadensprozesse an der Dammkrone ist jedenfalls mit den aufgezeigten messtechnischen Ausstattungen nicht möglich.

Die Dammkrone eignet sich als integraler Indikator für den Zustand des Absperrbauwerks in Extremsituationen. Sobald ein signifikanter Schaden an der Dammkrone feststellbar ist, besteht jedenfalls größte Gefahr für das Versagen des Bauwerkes (Aufleger und Lopez 2016). Durch ein kabelbasiertes System besteht die Möglichkeit, strukturelle Veränderungen der Dammkrone und sehr deutliche Deformationen bereits in einem frühen Stadium zu erkennen. Hierzu muss ein geeignetes Messverfahren verwendet werden, welches in der Lage ist, zu jeder Zeit eine Verletzung oder aber auch eine größere Deformation im Bereich des Kabels zu erkennen. Diese Information und gegebenenfalls auch der Ort einer Schadstelle ist dann an das Leitsystem bzw. an die zuständigen Personenkreise direkt weiterzuleiten. Durch die Integration 
eines solchen Dammbruchwarnsystems wäre es möglich, die tatsächliche Vorwarnzeit, d.h. die Zeitdifferenz zwischen dem zuverlässigen Erkennen des Problems an der Anlage und dem Eintreffen der Flutwelle im Bereich der kritischen Infrastrukturen, wirksam zu reduzieren (Abb. 9).

Der Einsatz kabelbasierter Systeme ist u. a. aus verschiedenen Anwendungen verteilter faseroptischer Messungen gut bekannt (z. B. Goltz et al. 2009), welche jedoch grundsätzlich der „normalen" Bauwerksüberwachung, insbesondere dem Erkennen von Durchsickerungen, aber auch in besonderen Fällen der verteilten Deformationsmessung dienen (Höpffner et al. 2012). Ein Dammbruchwarnsystem besteht aus einem geeigneten Kabel, welches im Bereich der Dammkrone in Längsrichtung innerhalb des Dammbauwerkes installiert wird (Abb. 8), und aus einem an einer günstigen und sicheren Position angeordneten sehr robusten Messsystem, welches ständige Messungen durchführt. Ein Ausfall dieses Systems resultiert in einer Warnmeldung.

\section{Die Menschen an der Talsperre}

Die Sicherheit einer jeden einzelnen Talsperre muss ab dem Zeitpunkt des Baubeginns zu jeder Zeit in einem dem Gefährdungspotenzial der Anlage entsprechenden sehr hohen Maße gewährleistet sein. Bedingt durch das immer höher werdende Alter der Talsperren verändern sich die bei der Überwachung der Anlagen angewandten Verfahren und Vorgehensweisen. Der Datenumfang wird zumindest an den größeren Talsperren immer höher werden. Die Digitalisierung und das Einbinden aller verfügbaren Informationen in übergeordnete DatenmanagementSysteme wird erhebliche Vorteile schaffen. Unersetzbar werden jedoch die Personen sein, welche sowohl bei den ständigen visuellen Kontrollen, bei der Bewertung der gesammelten Bauwerksdaten aber auch bei den immer wieder notwendigen grundsätzlichen Überprüfungen der Talsperren erhebliche Verantwortung tragen. Diese Aufgaben müssen von einer Generation an die nachfolgende weitergegeben werden. Die Talsperren von heute werden dann noch sehr lange sehr sicher stehen!

Funding Open access funding provided by University of Innsbruck and Medical University of Innsbruck.

Open Access Dieser Artikel wird unter der Creative Commons Namensnennung 4.0 International Lizenz (http:// creativecommons.org/licenses/by/4. $0 /$ deed.de) veröffentlicht, welche die Nutzung, Vervielfältigung, Bearbeitung, Verbreitung und Wiedergabe in jeglichem Medium und Format erlaubt, sofern Sie den/die ursprünglichen Autor(en) und die Quelle ordnungsgemäß nennen, einen Link zur Creative Commons Lizenz beifügen und angeben, ob Änderungen vorgenommen wurden.

\section{Literatur}

Aufleger, M. (2007): Der gute alte Erddruckgeber. In: Wasserbau an der TU München. Beiträge zum wasserbaulichen Symposium anlässlich des Abschieds von Herrn Univ.-Prof. Dr.-Ing. Theodor Strobl am 16. März 2007. München: Technische Universität München (= Berichte des Lehrstuhls und der Versuchsanstalt für Wasserbau und Wasserwirtschaft, 110), S. 11-17.

Aufleger, M. (2013): Mach's mit! - Oder besse ohne? - Grundablässe im internationalen Vergleich. In: WasserWirtschaft - Fachzeitschrift für Wasser und Umwelttechnik 05, S. 140-143. Springer Professional, Springer Fachmedien GmbH, Wiesbaden.

Aufleger, M., Lopez, D. (2016): Die Dammkrone als Indikator für die Talsperrensicherheit in Extremsituationen. In: WasserWirtschaft - Fach zeitschrift für Wasser und Umwelttechnik 106/6, S. 136-139. Springer Professional, Springer Fachmedien $\mathrm{GmbH}$, Wiesbaden.

DWA (2011): Merkblatt DWA-M 514 - Bauwerksüberwachung an Talsperren Regelwerk de Deutschen Vereinigung für Wasserwirtschaft, Abwasser und Abfall e.V. (DWA).
Fry, J.-J., Blais, J.-P., Perzlmaier, S., Aufleger, M., Goltz, M. (2007): Introduction to the Detection of Internal Erosion. In: Assessment of the Risk of Internal Erosion of Water Retaining Structures: Dams, Dykes and Levees. Intermediate Report of the European Working Group of ICOLD. München: Technische Universität München (= Berichte des Lehrstuhls und der Versuchsanstal für Wasserbau und Wasserwirtschaft, 114), ISBN 978-3-940476-04-3, S. 132-143.

Goltz, M., Etzer, T., Aufleger, M., Muckenthaler, P (2009): Assessing the Cricital Seepage Velocity Causing Transport of Fine Particles in Embankment Dams and Their Foundation. In: Bauer, Erich, Semprich, Stephan, Zenz, Gerald: Proceedings of the 2nd International Conference on Long Term Behaviour of Dams. 12th-13th October 2009, Graz. Graz: Verlag der Technischen Universität Graz, ISBN 978-3-85125-0701, S. 479-484.

Hoepffner, R., Kapeller, G., Goltz, M., Aufleger, M. (2012): Verteilte faseroptische Dehnungsmessungen in Betonbauwerken, Lockergestein und Hangrutschungen. In: WasserWirtschaft -
Fachzeitschrift für Wasser und Umwelttechnik 2012/09, S. 35-41.

International Commission on Large Dams (ICOLD) (2009): Bulletin 138 General approach to Dam Surveillance-Basic elements in a "dam safety" process. Paris

International Commission on Large Dams (ICOLD) (2014): Bulletin 158 Dam Surveillance Guide (Preprint). Paris.

Lang, M., Overhoff, G. (2016): Das neue Dichtungs- und Kontrollsystem am Sylvensteinspeicher. In: WasserWirtschaft - Fachzeitschrift fü Wasser und Umwelttechnik 2016/06, S. 36-39. Lopez, D., Aufleger, M. (2017): Assessment of an embankment dam break warning system based on historical dam failures. In: Noorzad, A. Bauer, E., Ghaemian, M., Ebrahimian, B. LTBD 2017. Long-term behaviour and environmentally friendly Rehabilitation Technologies of dams. Proceedings of the 4 th International Conference. 17th-19th October, 2017, Tehran, Iran. Graz: Verlag der Technischen Universität Graz, ISBN 978 3-85125-564-5, S. 58-64. 